\title{
Seguimento de 8 anos de prótese aórtica Medtronic-Hall: influência da anticoagulação oral na ocorrência de embolias
}

\author{
Iseu Affonso da COSTA*, Djalma Luiz FARACO*, Fábio SALLUM*, Elson OLIVEIRA*, Aldo PESARINI*, \\ Francisco Diniz Affonso da COSTA*.
}

RBCCV 44205-64

COSTA, I. A.; FARACO, D. L.; SALLUM, F.; OLIVEIRA, E. C.; PESARINI, A.; COSTA, F. D. A. - Seguimento de 8 anos de prótese aórtica Medtronic-Hall: influência da anticoagulação oral na ocorrência de embolias. Rev. Bras. Cir. Cardiovasc., 3(3): 175-181, 1988.

RESUMO: Foram estudados 165 sobreviventes à prótese aórtica Medtronic-Hall, operados entre setembro de 1979 e setembro de 1987. As idades variaram entre 14 e 68 anos ( $m=35,2) ; 129$ eram masculinos, 36 femininos. As lesōes operadas foram 70 insuficiências, 37 estenoses e 39 duplas lesōes aórticas. Havia 8 disfunçōes de próteses, 8 endocardites agudas e 3 comunicações interventriculares + insuficiência aórtica. O seguimento foi de $163(98,7 \%)$ pacientes, sendo 9 perdidos durante o período de observação. Houve 45 óbitos tardios, sendo de $59 \%$ EP $10,9 \%$ a probabilidade atuarial de sobrevida aos 8 anos. Houve 26 episódios embólicos em 21 pacientes, sendo de $69,8 \%$ EP $11,7 \%$ a probabilidade de não ocorrência de embolia e de $39,7 \%$ EP $10,4 \%$ de sobrevida sem embolia. A incidência global de episódios foi de 3,5 por pacientes/ano, sendo 6 fatais $(23 \%)$. Quanto ao uso de anticoagulação oral, os pacientes dividiram-se em 3 grupos. No grupo A houve 144 pacientes, com uma incidência linearizada de 3,2 por 100 pacientes/ano. No grupo B -21 pacientes que passaram a tomar anticoagulantes em seguida á operação, houve 1,9 episódios por 100 pacientes/ano. No grupo $\mathrm{C}-9$ pacientes que iniciaram a anticoagulaçẫo após a ocorrência de um episódio embólico, apresentaram 8,1 embolias por 100 pacientes/ano. Concluímos que não foi possível demonstrar a eficiência da anticoagulação oral, nas condiçōes em que foi conduzida, na presente série, na prevenção das embolias. Após a ocorrência de um episódio embólico, sua instituiçāo nāo foi suficiente para diminuir, significativamente, a recorrência desta complicação.

DESCRITORES: embolia, próteses valvulares cardiacas; anticoagulação, próteses valvulares cardiacas; próteses valvulares cardíacas, cirurgia.

\section{INTRODUÇÃO}

A prótese de disco pivotante Medtronic-Hall teve seu emprego clínico registrado, inicialmente, por Hall et alii ${ }^{7}$, em 1977. A partir de setembro de 1979 passamos a utilizá-la em posição aórtica.

\section{MATERIAL E MÉTODOS}

O presente trabalho baseia-se no seguimento de 165 pacientes sobreviventes à prótese aórtica Medtronic-Hall, isolada, operados entre setembro de 1979 e setembro de 1987.

Trabalho realizado no Departamento de Cirurgia da Universidade Federal do Paraná e na Santa Casa de Misericórdia de Curitiba. Curitiba, PR. Brasil.

Apresentado ao 15: Congresso Nacional de Cirurgia Cardiaca. Rio de Janeiro, RJ, 7 e 8 de abril, 1988.

- Da Santa Casa de Misericórdia de Curitiba e do Hospital de Clínicas da Universidade Federal do Paraná.

Endereço para separatas: Iseu Costa. Rua Carmelo Rangel, 1816, 80420 Curitiba, PR, Brasil. 
COSTA, I. A.; FARACO; D. L.; SALlUM, F.; OlIVEIRA, E. C.; PESARINI, A.; COSTA, F. D. A. - Seguimento de 8 anos de prótese aórtica Medtronic-Hall: influência da anticoagulação oral na ocorrência de embolias. Rev. Bras. Cir. Cardiovasc., 3(3): 175-181, 1988.

As operaçōes foram realizadas na Santa Casa de Misericórdia de Curitiba e no Hospital de Clínicas da Universidade Federal do Paraná. Cento e vinte e nove pacientes eram masculinos e 36 femininos. A idade variou de 14 a 68 anos (média 35,2 anos).

A Tabela 1 indica os diagnósticos dos casos operados.

TABELA 1

DIAGNOSTICO DOS 165 PACIENTES COM PRÓTESE AORTICA MEDTRONIC-HALL

\begin{tabular}{lc}
\hline \multicolumn{1}{c}{ DIAGNÓSTICO } & $N^{\circ}$ \\
\hline Insuficiência Aórtica & 70 \\
Estenose Aórtica & 37 \\
Dupla Lesão Aórtica & 39 \\
Disfunção de Prótese & 08 \\
Endocardite Aguda & 08 \\
$\begin{array}{l}\text { Comunicação Interventricular }+ \\
\text { Insuficiência Aórtica }\end{array}$ & 03 \\
\hline \multicolumn{1}{c}{ Total } & 165 \\
\hline
\end{tabular}

Estudamos os 165 pacientes que tiveraram alta hospitalar após a operação. Cento e sessenta e três pacientes $(98,8 \%)$ foram acompanhados de um semestre a 8 anos. e 9 pacientes foram perdidos de seguimento, em tempos variáveis após a operaçāo, tendo sido computados nos cálculos de freqüēncia das ocorrências, de acordo com as regras atuariais.

As operaçōes foram realizadas com as técnicas usuais de circulação extracorpórea, utilizando-se oxigenanadores de bolhas sob hipotermia moderada $\left(28^{\circ}-30^{\circ} \mathrm{C}\right)$.

A proteção miocárdica foi obtida com solução cardioplégica cristalóide.

Após a alta hospitalar, os pacientes foram seguidos ativamente em ambulatório, em contato com os médicos assistentes ou por telefone com os próprios pacintes, a cada 6 meses. Procuramos ser o mais estrito possivel, considerando como episódio embólico todo fenômeno isquêmico do sistema nervoso central ou periférico transitório ou permanente, documentado ou referido pelos pacientes.

Cento e quarenta e quatro pacicentes não fizeram uso de anticoagulantes orais, tendo sido aconselhado o uso de ácido acetilsalicílico na dose de $500 \mathrm{mg}$ diários.

Vinte e um pacientes tiveram regime de anticoagulação oral instituído após a intervençāo. Utilizou-se droga cumarínica (marcumar) procurando-se manter o tempo de atividade de protrombina em torno de $25 \%$ do valor controle.

Nove pacientes passaram a receber o cumarinico após a ocorrência de um episódio embólico.

A decisão sobre o uso de anticoagulantes foi tomada, juntamente com os pacientes e seus médicos assistentes, de acordo com as condiçōes sócio-econômicas e a possibilidade de controle laboratorial.

As probabilidades de ocorrência dos eventos foram calculadas segundo a metodologia de STARR \& GRUNKEMEIR ${ }^{14}$.

A siginificância das diferenças nos vários subgrupos foi calculada utilizando-se o teste $Z$ entre duas proporções populacionais ${ }^{8}$.

\section{RESULTADOS}

Foi possível realizar o seguimento de 163 pacientes $(98,7 \%)$ tendo sido perdidos de controle, em tempos variáveis após a operação, 9 pacientes. O seguimento total perfez 732 pacientes/ano.

Houve 45 óbitos tardios, sendo de 59\% EP 5,3\% a probabilidade de sobrevida após 8 anos (Gráfico 1).

Observaram-se 26 episódios embólicos em 21 pacientes, sendo a incidência linearizada de embolias de $3,5 \%$ pacientes/ano.

Dos 144 pacientes que não tomaram anticoagulantes orais após a operação, $19(13,1 \%)$ apresentaram embolias.

Dos 21 pacientes que passaram a tomar anticoagulantes após a operaçāo, 2 apresentaram embolia $(9,5 \%)$.

Nove outros pacientes passaram a tomar anticoagulantes somente após a ocorrência de 1 episódio embó-

\section{GRÁFICO 1}

PRÓTESE AÓRTICA MEDTRONIC-HALL

PROBABILIDADE DE SOBREVIDA - 165 PACIENTES

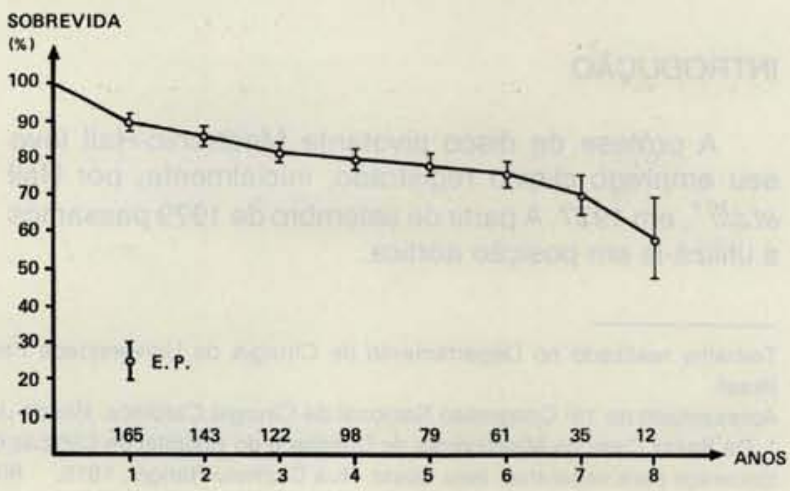


COSTA, I. A.; FARACO, D. L.; SALLUM, F.; OLIVEIRA, E. C.; PESARINI, A.; COSTA, F. D. A. - Seguimento de 8 anos de prótese aórtica Medtronic-Hall: influência da anticoagulação oral na ocorrência de embolias. Rev. Bras. Cir. Cardiovasc., 3(3): 175-181, 1988.

lico, 3 dos quais apresentaram recidiva de embolia $(33,3 \%)$.

O teste $Z$ aplicado a estes 9 subgrupos não revelou significância nas diferenças de incidēncia de embolias entre eles.

A possibilidade atuarial de não ocorrência de episódios embólicos foi de $69,5 \%$ EP $11,9 \%$ e a probabilidade cumulativa de sobrevida sem embolia foi de $38 \%$ EP 10,5\%, em 8 anos (Gráficos 2 e 3).

GRÁFICO 2

PRÓTESE AORTICA MEDTRONIC-HALL PROBABILIDADE DE NATO OCORRENNCIA DE EMBOLIA 165 PACIENTES



GRÁFICO 3

PRÓTESE AORTICA MEDTRONIC-HALL PROBABILIDADE DE SOBREVIDA SEM EMBOLIA 165 PACIENTES

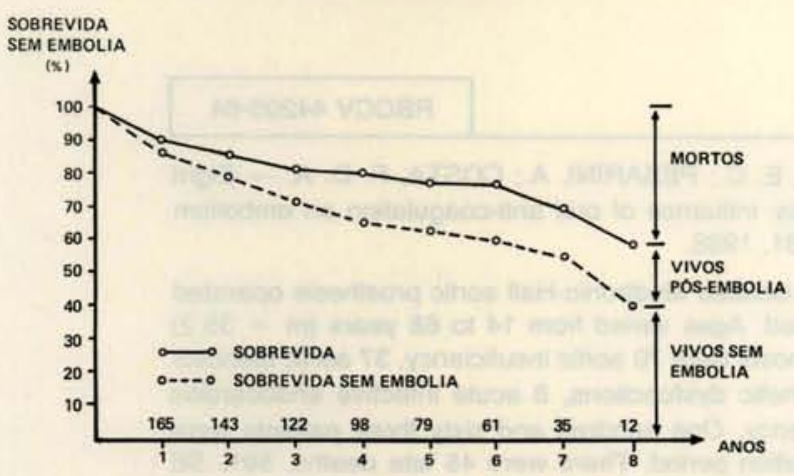

Analisando separadamente os subgrupos, conforme o uso de anticoagulantes, verificamos que:

Os pacientes que nāo tomaram anticoagulantes após a operação apresentaram incidência de 3,2\% episódios por 100 pacientes/ano.

Os 21 pacientes que tomaram anticoagulantes a partir da operação apresentaram uma incidência linearizada de 1,9 episódios por 100 pacientes/ano.

Considerando, separadamente, o grupo de 9 pacientes que passaram a tomar anticoagulantes após a ocorrência de um episódio embólico, verificamos que a incidência foi de 8,1 episódios por 100 pacientes/ano.

2.

A distribuição das embolias está indicada na Tabela

TABELA 2

DISTRIBUICAAO DAS EMBOLIAS

165 PRÓTESES AORTICAS MEDTRONIC-HALL

Sistema Nervoso Central

16

Acidentes Isquêmicos Transitórios

05

Membro Inferior

05

Membro Superior

01

Coronariano

01

\section{DISCUSSÃO}

A comparação do desempenho de próteses valvulares cardíacas apresenta notórias dificuldades. A grande maioria das série publicadas de próteses mecânicas tem sido com uso de anticoagulantes orais. Problemas de tromboembolismo e hemorragia continuam a afetar os portadores de próteses cardíacas mecânicas e respondem por cerca de $75 \%$ de suas complicações.

Há dados conflitantes, de centros diferentes, sobre a eficácia da anticoagulação oral, na prevenção dos acidentes embólicos ${ }^{6,12,15}$.

Entretanto, para a maioria ${ }^{1.4}$, o uso de anticoagulantes após o implante de próteses mecânicas é considerado mandatório.

Tem sido registrada a eficácia de tratamentos anticoagulantes bem padronizados e controlados.

Face às dificuldades logísticas em manter os pacientes em condições próximas do ideal de controle de anticoagulantes, regimes menos intensos e a combinação com agentes inibidores da adesividade plaquetária tẽm sido propostos, mas a avaliação definitiva de seu valor não está claramente estabelecida.

Em 1982, publicamos observaçōes sobre o seguimento inicial de próteses aórticas Medtronic-Hall sem anticoagulantes ${ }^{2}$.

A observaçāo de incidência relativamente elevada ( $5,2 \%$ por paciente/ano) de embolias nos levou a recomendar o uso de anticoagulantes orais, sempre que haja condições de um controle seguro de atividade protrombínica, ou que tenha ocorrido uma embolia. Nestas condiçōes, foram formados os subgrupos que compōem a presente série. 
COSTA, I. A.; FARACO, D. L.; SALLUM, F.; OLIVEIRA, E. C.; PESARINI, A.; COSTA. F. D. A. - Seguimento de 8 anos de prótese aórtica Medtronic-Hall: influência da anticoagulação oral na ocorrência de embolias. Rev. Bras. Cir. Cardiovasc. 3(3): 175-181, 1988.

Devemos reconhecer que năo foi possivel conduzir a anticoagulaçāo em condiçōes ideais de controle clínico e laboratorial.

Por outro lado, a prescrição de ácido acetilsalicilico dificilmente é controlável, e a maioria de nossos pacientes faz uso sem rigorosa regularidade.

Cabe, aos centros de cirurgia cardiaca que empregam próteses valvulares, o seguimento cuidadoso dos pacientes, para chegar a uma avaliação objetiva de seus próprios resultados, face às muitas variáveis que influem na evoluçẳo e desempenho destas próteses ${ }^{9}$.

Tais variáveis incluem as relativas aos pacientes, tais como área cardiaca, fibrilação atrial, idade, classe funcional, anticoagulação, nível sócio-econômico e distribuição geográfica; e as relativas à prótese em si mes-

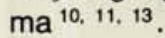

Todas estas variaçōes podem influir na incidência de episódios tromboembólicos, acrescentando-se, neste particular, a variabilidade dos critérios de definição e registro destas complicações.

GERSH et alii ${ }^{5}$, revendo numerosos trabalhos sobre a avaliaçāo clínica de próteses valvulares, estimaram em 0,5 e 3,0 episódios por 100 pacientes/ano a incidência de embolias em próteses aórticas.

Estes mesmos autores sugeriram que a liberação de uma nova prótese deveria exigir que a incidência dos eventos pós-operatórios não exceda o dobro do observado com as válvulas aceitas correntemente.

A mortalidade tardia e a incidência global de episódios embólicos nos pacientes com a prótese aórtica Medtronic-Hall da presente série estão enquadradas dentro daqueles limites. Faz exceção o subgrupo dos pacientes seguidos após a ocorrência de um episódio embólico. Neles, a incidência de nova embolia foi de 8,1 episódios por 100 pacientes/ano, que supera o limite proposto de $2 \times 3,0$ episódios por 100 pacientes/ano. Embora o subgrupo seja pequeno, e seguido por tempo relativamente curto, cremos que se caracteriza como uma coorte de alto risco de recorrẽncia de embolia.

A comparação da incidência de embolias entre os grupos com e sem anticoagulação não permitiu demonstrar sua eficácia na diminuição do número de acidentes.

Note-se, entretanto, que o grupo anticoagulado apresenta um total reduzido de pacientes.

Vários autores ${ }^{4}$ têm assinalado que a ocorrência de um episódio embólico aumenta de até 4 vezes a possibilidade de uma nova embolia. O início da anticoagulação após a ocorrência de uma embolia não foi capaz de diminuir o risco de recidiva.

Este fato milita a favor da idéia de substituir uma prótese mecânica após o primeiro episódio embólico, possivelmente por uma bioprótese.

RBCCV $44205-64$

COSTA, I. A.; FARACO, D. L.; SALlum, F.; OliVeIRA, E. C.; PESARINI, A.; COSTA, F. D. A. - Eight years follow-up of the Medtronic-Hall aortic prosthesis: influence of oral anti-coagulation on embolism incidence. Rev. Bras. Cir. Cardiovasc., 3(3): 175-181, 1988.

ABSTRACT: One hundred and sixty five survivors of isolated Medtronic-Hall aortic prosthesis operated on from September 1979 to September 1987 were studied. Ages varied from 14 to 68 years $(\mathrm{m}=35.2)$ and 129 patients were male, 36 female. Preoperative diagnosis were 70 aortic insuficiency, 37 aortic stenosis and 39 double lesions. There were additionally 8 prosthetic dysfunctions, 8 acute infective endocarditis and 3 interventricular septal defects plus aortic insufficiency. One hundred and sixty three patients were followed (98.72), 9 of them being lost during the observation period. There were 45 late deaths, $59 \%$ SE $10.9 \%$ being the actuarial survival probality in 8 years. Twenty one patients suffered 26 embolic episodes, 69.8 SE $11.7 \%$ the probability of freedom from embolism and $39.7 \%$ SE $10.4 \%$ the chance of survival free from embolism. The rate of embolism episodes was $3,5 \%$ per patients/year in the entire series, 6 of them being lethal. In relation to the use of oral anticoagulation patients were divided into three sub-groups. Sub-group A included 144 patients, with a linearized incidence of $3.2 \%$ episodes per patients/year. Sub-group $B$ included 21 patients who used anticoagulants after surgery, with an incidence of $1.9 \%$ per patients/year. Sub-group $C$ comprised 9 patients who were put on anticoagulants after the occurence of an embolic episode. This sub-group presented 8.1 episodes per patients/year. It is concluded that it was not possible to doccument the influence of anticoagulation in the conditions prevailing during the observation of this series. After the occurence of one embolic episode the institution of oral anticoagulation was not effective in decreasing chance of its reccurence.

DESCRIPTORS: embolism, heart valves prostheses; anticoagulation, heart valves prostheses; heart valves prostheses, surgery. 
COSTA, I. A.; FARACO, D. L.; SALlUM, F.; OlIVEIRA, E. C.; PESARINI, A.; COSTA, F. D. A. - Seguimento de 8 anos de prótese aórtica Medtronic-Hall: influência da anticoagulação oral na ocorrência de embolias. Rev. Bras. Cir. Cardiovasc., 3(3): 175-181, 1988.

\section{REFERÊNCIAS BIBLIOGRÁFICAS}

1 BLOOM, A. L. - The need for standartization of anticoagulant management. International Heart Valve Replacement Symposium. Lanzarote, Ilhas Canárias, Espanha, 1987.

2. COSTA, I. A.; FARACO, D. L.; SALLUM, F.; OLIVEIRA, E. C.; PESARINI, A.; QUINTANEIRO, V.; COSTA, F. D. A. - Evolução clínica da prótese de Hall-Kaster sem uso de anticoagulantes. Arq. Bras. Cardiol. 39(4): 229-233, 1982.

3. EDMUNDS Jr., L. H. - Thrombotic and bleeding complications of prosthetic heart valves. Ann. Thorac. Surg., 44(4): 430-445, 1987.

4. EDMUNDS Jr., L. H. - What's new cardiac surgery. Bul. Am. Coll. Surg. 73(1): 5-10, 1988.

5. GERSH, B. J.; FISHER, L. D.; SCHAFF, H. V.; RAHIMTOOLA, S. H.; REEDER, G. S.; FRATER, R. W. M. Mc GOON, D. C. - Issues concerning the clinical evaluation of new prosthetic valves. J. Thorac. Cardiovasc. Surg. 9(3): 460-466, 1986.

6. GÖSSINGER, H.; NIESSNER, H.; GRUBECK, B.; MŐSSLACHER, H.; BETTELHEIM, P.; MICZOCHS - DOMANIG, C. - Thromboembolism in patients with prosthetic heart valves: an adequately controlled intense anticoagulant therapy and its influence on the occurrence of thromboembolism in relátion to valve type. Thorac. Cardiovasc. Surg., 34(5): 283-86, 1985.

7. HALL, K. V.; FRYSAKER, I.; ROSTAD, H.; RESCH, F.; LILLEASEN P.; ENGE, L.; NITTER-HANGE, S. - The Hall-Kaster valve: the primary clinical experience. The Asscociation for Thoracic and Cardiovascular Surgery Annual Metting. Aarhus, Dinamarca, outubro 1977.

8. KAZMIER, L. J. - Estatística aplicada a economia e administração São Paulo, Mac Grow Hill, 1982.

9. KINSLEY, R. H.; ANTUNES, M. J.; COLSEN, P. R. St. Jude edical valve replacement. J. Thorac. Cardiovasc. Surg.,92(3 Pt1): 349-360, 1986.

10. MITCHEL, R. S.; MILLER, D. C.; STINSON, E. B.; JAMIESON, S. W.; BALDWIN, J. C.; SHUMWAY, N. E. Significant patient: related determinants of prosthetic valve performance. J. Thorac. Cardiovasc. Surg., 91(5): 803-817, 1986.

11. MURPHY, G. S. \& KLOSTER, O. - Late results of valve replacement surgery. II. Complications of prosthetic heart valves. Modern Conc. Cardiovasc. Dis., 48(11): 59-66, 1979

12. PASS, H. I.; SADE, R. M.; CRAWFORD, F. A.; HOLM, A. R. - Cardiac valve prostheses in children without antioagulation. J. Thorac. Cardiovas. Surg., 87(6): 832-835, 1984

13. PUMPHREY, C. W.; FUSTER, V.; CHESEBORO, J. H. - Sistemic thromboembolism in valvular heart disea- ses and prosthetic heart valves. Modern Conc. Cardiovas. Dis., 51(12):131-136, 1982.

14. STARR, A. \& GRUNKEMEIER, G. L. - Data base and methodology. In: LEFRAK, E. A \& STARR, A. - Cardiac valve prostheses. New York, Appleton-Century, 1979 .

15. THEVENET, A. - Lillehei-Kaster prosthesis in aortic position with and without anticoagulants. J. Cardiovasc. Surg., 21(6): 669-674, 1980 .

16. THOMSEN, P. B.; \& ALSTRUP, P. - Thromboembolism in patients without anticoagulants after aortic valve replacement with Lillehei-Kaster disc valve. Thorac. Cadiovasc. Surg., 27(5): 313-315, 1979.

AGRADECIMENTO: Agradecemos à Srta. Salete Pelanda a análise estatística de nossos dados.

\section{Discussão}

\section{DR. ANTOONIO CARLOS PENNA \\ Marilia, $S P$}

O alcance dos nossos comentários está limitado por 2 fatores: em primeiro lugar, por estarem baseados apenas no resumo do trabalho e não no trabalho original e, em segundo lugar, recebermos os resumos a menos de um mês do início do Congresso. (Slide) Limitados, então, pelo fator tempo, conseguimos atualizar os dados de apenas 39 de nossos pacientes que são portadores dessa prótese na posiçāo aórtica. As diversas patologias estão aqui discriminadas, a média de idade foi de 40 anos, a maior parte dos pacientes era do sexo masculino e o acompanhadmento máximo foi a 7 anos. (Slide) A comparação dos nossos resultados com os do Dr. Iseu mostra, quanto ao óbito tardio, uma pequena diferença com o trabalho apresentado, com mortalidade tardia de $29 \%$ e nós, com $24 \%$. Quanto aos episódios tromboembólicos, $13,6 \%$ dos pacientes do $\mathrm{Dr}$. Iseu os apresentaram, com mortalidade de $28,5 \%$ : os nossos números foram um pouco melhores $(10,2 \%)$, mas com mortalidade de $50 \%$, apesar do pequeno número de pacientes. Um dado que nos parece interessante e nos preocupa é que cerca de $1 / 3(33,3 \%)$ dos nossos pacientes apresentou um ou mais episódios hemorrágicos em conseqüência do uso de anticoagulantes, felizmente nenhum fatal. (Slide) Comparando os dados do Dr. Iseu com os da literatura internacional, selecionamos estes 3 trabalhos: o de Nitter-Hauge, do grupo do próprio Dr. Hall, com um número grande de pacientes operados (482), acompanhados até 5 anos; o de Starek, de 1983, com 140 pacientes acompanhados até 4,5 anos; e o Beaudet. 
COSTA, I. A.; FARACO, D. L.; SALlUM, F.; OliveIRA, E. C.; PESARINI, A.; COSTA, F. D. A. - Seguimento de 8 anos de prótese aórtica Medtronic-Hall: influência da anticoagulação oral na ocorrência de embolias. Rev. Bras. Cir. Cardiovasc., 3(3): 175-181, 1988.

o mais recente, de 1986 , com 154 pacientes acompanhados, aproximadamente no mesmo período de tempo que os do $\mathrm{Dr}$. Iseu. A sobrevida dos pacientes destes autores é significantemente melhor do que a apresentada pelo Dr. Iseu, mas estes autores não apresentam o grau funcional pré-operatório dos seus doentes, o que pode significar que o $\mathrm{Dr}$. Iseu está operando um grupo bastante grave de pacientes. O número de episódios de tromboembolismo que apresentam, evidenciados pelo número de eventos por 100 pacientes/ano, também é bastante melhor do que os apresentados pelo $\mathrm{Dr}$. Iseu. No entanto, é preciso fazerem-se duas ressalvas muito importantes: todos os autores anticoagulam sistematicamente os seus pacientes, o que não acontece com todos os pacientes aqui apresentados e, em segundo lugar, se considerarmos apenas os pacientes do Dr. Iseu que tomaram anticoagulante logo após a cirurgia, este número cai, significativamente, para 1,9 eventos/100 pacientes/ano, número perfeitamente compatível com os da literatura internacional. O mesmo raciocínio pode ser feito para a porcentagem de pacientes livres de tromboembolismo ao fim de um certo número de anos. Finalizando, o grande valor do trabalho do Dr. Iseu é evidenciar, mais uma vez, que prótese metálica, qualquer que seja ela e em qualquer posição, é igual à anticoagulação. Os nossos cumprimentos ao Dr. Iseu e equipe, pelo elevado número de pacientes operados, pelo longo tempo de acompanhamento e pelos resultados obtidos, que nos parecem perfeitamente compativeis com os da literatura mundial.

\section{DR. JARBAS DINKHUYSEN}

São Paulo, SP

O nosso Serviço nāo usa a prótese Medtronic-Hall. A prótese mecânica de escolha para posição aórtica é a Starr-Edwards e, quando a indicação recai para próteses de baixo perfil, temos empregado a Omniscience e a Omnicarbon. Por este motivo, não apresentaremos números de nossa casuística. O primeiro comentário diz respeito ao trabalho apenas referir a incidência de tromboembolismo em pacientes portadores de prótese mecânica, deixando de enfocar seu corolário imediato: os problemas hemorrágicos. $\mathrm{O}$ fiel da balança entre tromboembolismo e acidentes hemorrágicos, excluídas causas diversas, é, sem dúvida, o método de anticoagulaçāo empregado, as associações medicamentosas, o controle laboratorial, etc. Desta maneira, se ocorrer anticoagulação insuficiente ou em excesso, a incidência de tromboembolismo ou de problemas hemorrágicos será maior. O slide demonstra esta realidade, num grupo de pacientes submetidos a substituição aórtica por próteses diversas. Beaudet e colaboradores (1986) publicavam um artigo com uma casuistica de prótese Medtronic-Hall em posição aórtica praticamente superponivel à apresentada pelo autor. Observa-se que os índices de sobrevida atuarial (slide) e de probabilidade de ocorrência de embo- lia (slide) são significativamente superiores aos aqui apresentados. Em relação ao número de episódios tromboembólicos, comparamos o Grupo A (slide) (pacientes sem anticoagulantes) do autor com grupo também nāo anticoagulados com próteses Medtronic-Hall, Starr-Edwards e St. Jude Medical, observando-se indices de episódios tromboembólicos bastante superiores aos apresentados pelo autor. Já com o Grupo B, isto é, pacientes anticoagulados após a operação, não se observaram diferenças significativas. Quanto à incidência global de episódios embólicos, incluindo os três grupos (A, B e C) (slide), comparamos com um grupo de pacientes operados e controlados pelo Dr. Braile (São José do Rio Preto), dos quais $70 \%$ dos pacientes usam apenas aspirina + dipiridamol e $30 \%$ sāo anticoagulados, observando-se indices semelhantes. Perguntaria se os pacientes do Grupo C, isto é, aqueles que passaram a ser anticoagulados após o acidente tromboembólico, não pertencem ao Grupo A e por que há uma incidência tão elevada de episódios embólicos neste grupo, visto que, após o primeiro acidente, os pacientes foram anticoagulados? Para encerrar (slide), gostaria de dizer que a análise do binômio tromboembolismo/hemorragia, em pacientes anticoagulados e com próteses valvulares cardiacas, é multifatorial, dependendo de inúmeros variáveis relativos às características individuais da coagulação de cada paciente, do quadro hemodinâmico da patologia a ser tratada, do tipo de prótese empregada, dos tipos de medicamentos empregados e suas interaçōes, de eventuais patologias associadas, da maneira como a anticoagulação é conduzida e, naturalmente, de fatores geográficos e sócio-econômicos dos pacientes. Cumprimento o Dr. Iseu e seus colaboradores, pelo excelente trabalho apresentado. Muito obrigado.

\section{DR. ISEU COSTA \\ (Encerrando)}

Agradeço ao Dr. Penna, por seus comentários. Parece-me, à primeira vista, que os dados da estatística do Dr. Penna não diferem, significativamente, dos nossos, se levarmos em conta os limites de confiança necessariamente mais amplos em série com um número relativamente pequeno, como a analisada pelo $\mathrm{Dr}$. Penna, de 39 pacientes. Obviamente, concordamos com a importância atribuída aos acidentes hemorrágicos da anticoagulação. Em nossa série, apenas 21 pacientes usaram, sistematicamente, cumarínicos a partir da operação; outros 9 somente após a ocorrência de embolia. Portanto, os subgrupos são pequenos e, embora os acidentes hemorrágicos tenham sido raros, não nos animamos a tirar conclusões que possam ser válidas. De qualquer forma, parece que, quanto maior o esforço em manter uma anticoagulação efetiva, maior será a ocorrência de acidentes hemorrágicos. Quanto à comparação com séries de outros autores, concordamos com a opinião de que, no seguimento das próteses, a importância das variáveis relacionadas com os pacientes é, por vezes, 
COSTA, I. A.; FARACO, D. L.; SALlUM, F.; OLIVEIRA, E. C.; PESARINI, A.; COSTA, F. D. A. - Seguimento de 8 anos de prótese aórtica Medtronic-Hall: influência da anticoagulação oral na ocorrência de embolias. Rev. Bras. Cir. Cardiovasc., 3(3): 175-181, 1988.

maior do que a do próprio tipo de prótese empregada. Agradeço, também, ao Dr. Dinkhuysen, seus comentários, que voltaram a enfocar a incidência de embolias com e sem anticoagulantes e com vários tipos de prótese. O objetivo de nosso trabalho foi verificar que magnitude devemos esperar na incidência de embolias, quan- do usamos próteses em pacientes nos quais é virtualmente impossivel manter uma anticoagulação bem controlada. Creio que nossos dados demonstram que, embora a incidência seja claramente maior quando não usamos anticoagulantes, ela situa-se em níveis não catastróficos. Obrigado. 